\title{
Yeast expression vectors using RNA polymerase III promoters
}

(Shuttle plasmids; RPR1; tRNA gene; anti-sense RNA; ribozymes)

\author{
Paul D. Good and David R. Engelke \\ Department of Biological Chemistry, University of Michigan, Ann Arbor, MI 48109-0606, USA \\ Received by G.P. Livi: 14 March 1994; Revised/Accepted: 22 June/7 July 1994; Received at publishers: 2 September 1994
}

\section{SUMMARY}

A series of Saccharomyces cerevisiae - Escherichia coli shuttle vectors is described in which small RNAs can be stably expressed in yeast from two different promoters for RNA polymerase III transcription. The vectors are available in either high- or low-copy-number forms with either URA3, HIS3, or TRP1 selection markers, and are based on a previously described set of plasmid vectors [Sikorski and Hieter, Genetics 122 (1989) 19-27]. Transcripts have structured pre-tRNA or RPR1 leaders fused to RNA corresponding to inserted sequences. Levels of RNA accumulation are dependent on plasmid copy number and the type of transcript.

\section{INTRODUCTION}

There are a number of vector systems for expression of proteins in Saccharomyces cerevisiae from different promoters for RNA polymerase-II-mediated transcription. For some purposes, however, it could be advantageous to express high levels of RNA in a way that avoids shunting of transcripts into a pre-mRNA pathway while at the same time stabilizing the transcripts to promote their accumulation. Examples of potential uses might be expression of antisense RNAs, ribozymes, or 'decoy' structured RNA motifs.

Correspondence to: Dr. D.R. Engelke, Department of Biological Chemistry, 5413 Medical Science I, University of Michigan, $1301 \mathrm{E}$. Catherine Street, Ann Arbor, MI 48109-0606, USA. Tel. (1-313) 763-0641; Fax (1-313) 763-4581;

e-mail: david.r.engelke@um.cc.umich.edu

Abbreviations: Ap, ampicillin; bp, base pair(s); $C E N / A R S$, centromere/ autonomous replication sequence; E., Escherichia, HIS3, S. cerevisiae gene encoding imidazoleglycerol-P dehydratase; $n t$, nuclentide(s); oligo, oligodeoxyribonucleotide; ori, origin of DNA replication; pol III, RNA polymerase III; $R P R 1, S$. cerevisiae gene encoding RNA subunit of ribonuclease P; S., Saccharomyces; SUP4, S. cerevisiae gene encoding ochre suppressor tyrosyl tRNA; TRP1, S. cerevissiae gene encoding $\mathrm{R}$-anthranilate isomerase; $U R A 3, S$. cerevisiae gene encoding orotidine5 '-phosphate decarboxylase.
Two types of pol III promoter have been used in this study to synthesize small RNAs in yeast. The first utilizes the SUP4 tRNA gene intragenic promoter (Kurjan et al., 1980; Koski et al., 1980) to obtain transcripts where the full tRNA primary transcript is fused to the $5^{\prime}$ end of the RNA to be expressed. This mimics naturally occuring dimeric tRNA genes in yeast, where the promoter is contained in the coding region of the first gene in the pair (Schmidt et al., 1980; Kjellin-Straby et al., 1984; Reyes et al., 1986; Straby, 1988). Without the processing signal of the second tRNA sequence, however, the leading tRNA tends to remain attached to the following RNA and might stabilize it against $5^{\prime}-3^{\prime}$ exonucleolytic attack. Similar approaches have already been described for using tRNAdriven antisense RNA and ribozymes in vertebrates (Adeniyi-Jones et al., 1984; Cotten and Birnstiel, 1989; Yu et al., 1993).

As a second strategy the intragenic promoter from the $S$. cerevisiae RNase P RNA gene ( $R P R 1)$ was used. This promoter is of the same class as that of tRNA genes, consisting primarily of an A box and B box within the transcribed $5^{\prime}$ leader (Lee et al., 1991b), but the structured $84 \mathrm{nt}$ leader is normally removed from the RNase P RNA 'mature' domain and destroyed (Lee et al., 1991a). However, fusion of the leader to RNAs that are not recog- 
nized in RNase $\mathrm{P}$ maturation leads to stable accumulation (Pagan-Ramos et al., 1994). In the present work we show that up to several thousand transcripts per cell accumulate from the TRNA gene and RPR1 gene promoters, depending on the copy number of the vector and the RNA expressed. A series of expression vectors is described for using these promoters in a variety of yeast genetic backgrounds.

\section{EXPERIMENTAL AND DISCUSSION}

\section{(a) Construction of the expression vectors}

The S. cerevisiae-E. coli shuttle vectors for pol III expression are termed the pIIIEx series and were derived from the pRS vectors of Sikorski and Hieter (1989). The general construction of the vectors is summarized in Fig. 1. In addition to an E. coli ori and $\mathrm{Ap}^{\mathrm{R}}$ marker, the plasmids contain either a $2 \mu$ ori for high-copy propagation in yeast or a $C E N / A R S$ sequence for maintenance of the plasmid in low-copy. Versions of these plasmids are available with $H I S 3, T R P 1$ or $U R A 3$ yeast selection markers. A complete listing of the available plasmid constructs is shown in Table I. The pol III-mediated expression constructs were inserted between the BamHI and Sall sites in the pRS plasmids. Promoter and terminator regions were amplified with appropriate restriction sites for cloning using the polymerase chain reaction, and clones were sequenced (Tabor and Richardson, 1987) to ensure fidelity. The exact sequences of the promoter/terminator regions, along with the predicted structures of the primary transcripts, are shown in Fig. 2.

All plasmids contain the pol III terminator sequence from the $R P R 1$ gene from an $E c o R I$ site introduced at the end of the mature coding region to $96 \mathrm{bp}$ downstream (Lee et al., 1991a). Transcription termination occurs at the fifth or sixth $\mathrm{T}$ of the indicated sequence in Fig. 2. Vectors employing the SUP4 tRNA gene promoter (Goodman et al., 1977) contain SUP4 sequences from $77 \mathrm{bp}$ upstream from the mature tRNA coding region to 2 bp past the $3^{\prime}$ end of the mature tRNA coding region. At this point an $E c o$ RI restriction site is introduced and $S U P 4$ fragment is joined to the RPR 1 terminator. Vectors using the $R P R 1$ leader promoter contain a fragment from 407 bp upstream to an EcoRI site introduced at the end of the 84-bp region encoding the leader of the RPR1 primary transcript. As depicted in Fig. 2, both the tRNAcontaining and $R P R 1$ leader-containing primary transcripts are predicted to be highly structured.

The $R P R 1$ terminator region of primary transcripts is predicted to be less structured, and is accessible to multiple nucleolytic activities in its removal from normal $R P R 1$ primary transcripts (Lee et al., 1991a). To test
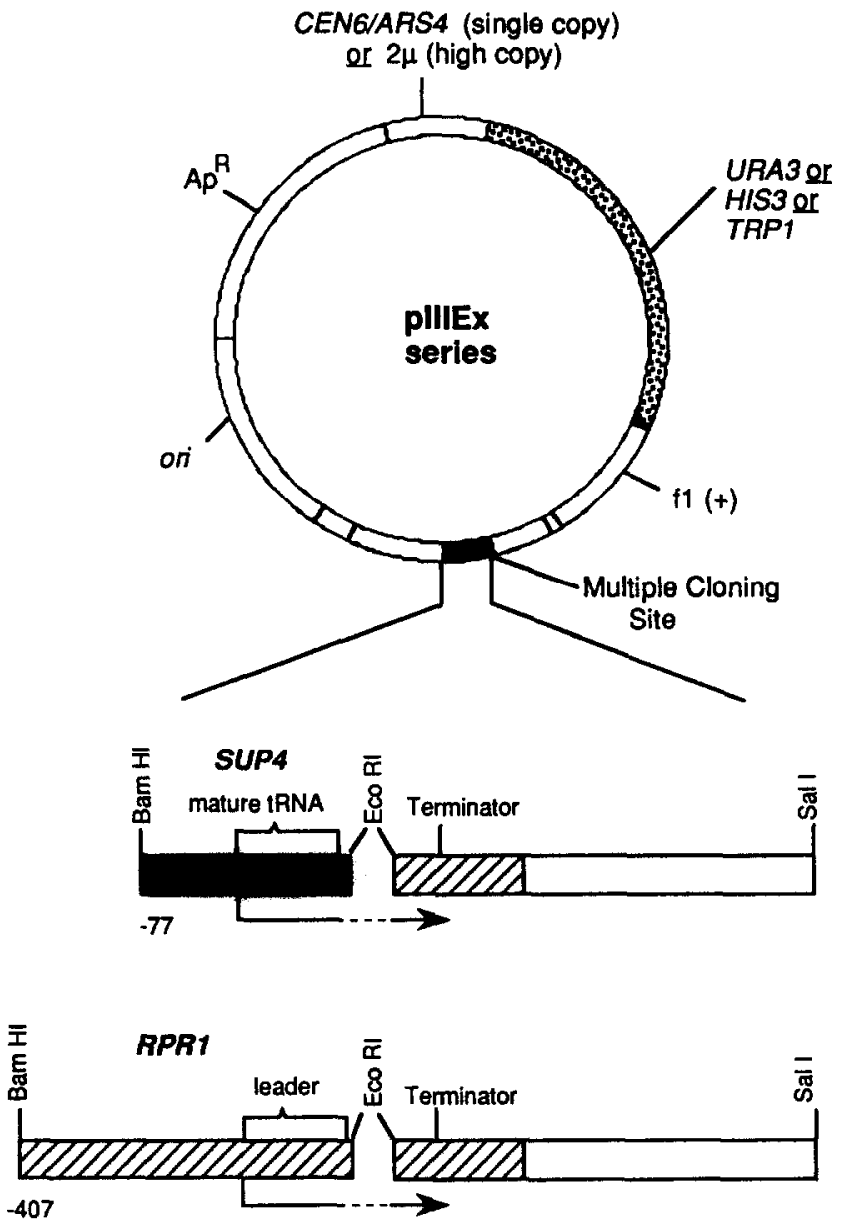

Fig. 1. General structure of the pIIIEx vectors. RNA polymerase III promoter/terminator constructs were inserted between the BamHI and SalI sites of several pRS shuttle vectors (Sikorski and Hieter, 1989). The pIIIEx 300 series have $C E N 6 / A R S 4$ sequences for low-copy maintenance and the the pIIIEx 400 series have $2 \mu$ ori for high-copy maintenance. Expression constructs have either the SUP4 tRNA intragenic promoter or the $R P R 1$ intragenic promoter followed by a unique $E c o \mathrm{RI}$ site for insertion of sequences to be expressed. All clones contain the $R P R 1$ poly $(\mathrm{T})$ terminator after the EcoRI site, although clones labelled with the suffix ' $/ 3$ 'ST' also have a strong stem/terminator inserted in front of the RPR1 terminator at the EcoRI site as described in section a. A complete list of the pIIIEx vectors by name, marker, copy number and types of pol III promoter and terminator is given in Table I.

whether transcripts might be more stable with tightly structured $3^{\prime}$ trailing sequences, we also created variants of the low-copy, HIS3 and $U R A 3$ marked expression vectors in which a strong stem followed immediately by a $\operatorname{poly}(\mathrm{T})\left(\mathrm{T}_{7}\right)$ terminator sequence was inserted immediately before the $R P R 1$ terminator. These variants have the sequence 5'-AATTCCGGACTTCGGTCCGGTTTTTTTAATT inserted at the EcoRI site, recreating the $E c o$ RI site only on the upstream side, and are referred to in Tables I and II with a ' 3 'ST' suffix. The inserted sequence is predicted to form a $\mathrm{G}+\mathrm{C}$-rich 6-bp stem with a tetraloop in the RNA, followed immediately by the poly(U) terminator. These $3^{\prime}$ stem constructs did not lead to higher levels of RNA transcript accumulation, but 
TABLE I

Features of available pIIIEx vectors

\begin{tabular}{|c|c|c|c|c|c|}
\hline Plasmid name ${ }^{a}$ & pRS parent $^{\mathrm{b}}$ & Marker $^{c}$ & $\begin{array}{l}\text { High or low } \\
\text { copyd }\end{array}$ & Promoter $^{e}$ & Terminator $\mathbf{f}$ \\
\hline pIIIEx313 tRNA & pRS313 & $H I S 3$ & low & SUP4 & RPR 1 \\
\hline pIIIEx 313 tRNA/3'ST & pRS313 & $H I S 3$ & low & SUP4 & 3 'Stem \\
\hline pIIIEx313 RPR & pRS313 & HIS3 & low & $R P R I$ & RPR1 \\
\hline pIIIEx313 RPR/3'ST & pRS313 & HIS3 & low & $R P R 1$ & 3 Stem \\
\hline pIIIEx423 tRNA & pRS423 & $H I S 3$ & high & SUP4 & $R P R I$ \\
\hline pIIIEx423 RPR & pRS423 & $H I S 3$ & high & $R P R I$ & $R P R 1$ \\
\hline pIIIEx314 tRNA & pRS314 & $T R P 1$ & low & $S U P 4$ & $R P R 1$ \\
\hline pIIIFx314 RPR & pRS314 & $T R P I$ & low & $R P R I$ & $R P R I$ \\
\hline pIIIEx424 tRNA & pRS424 & $T R P l$ & high & SUP4 & $R P R 1$ \\
\hline pIIIEx424 RPR & pRS424 & $T R P I$ & high & $R P R 1$ & $R P R 1$ \\
\hline pIIIEx316 IRNA & pR\$316 & $U R A 3$ & low & SUP4 & $R P R 1$ \\
\hline pIIIEx316 tRNA/3'ST & pRS316 & $U R A 3$ & low & $S U P 4$ & $3^{\prime}$ Stem \\
\hline pIIIEx316 RPR & pRS 316 & URA3 & low & $R P R 1$ & $R P R 1$ \\
\hline pIIIEx316 RPR/3'ST & pRS316 & $U R A 3$ & low & $R P R 1$ & $3^{\prime}$ Stem \\
\hline pIIIEx426 tRNA & pRS426 & $U R A 3$ & high & SUP4 & RPRI \\
\hline pIIIEx426 RPR & pRS426 & URA3 & high & $R P R 1$ & RPRI \\
\hline
\end{tabular}

a Plasmid names follow nomenclature for pRS series, also indicating which promoter is used.

${ }^{b}$ pRS plasmids are described in Sikorski and Hieter (1989).

c Yeast biosynthetic genes used to complement auxotrophies.

${ }^{d}$ Low-copy vectors have the $S$. cerevisiae CEN6/ARS4, high-copy vectors have the $2 \mu$ ori.

- The SUP4 tRNA gene and RPR1, RNase P RNA gene constructs are shown in Fig. 2.

${ }^{f}$ All vectors contain the RPR1 terminator, vectors designated 3'ST(3'Stem) have an additional hairpin stem and terminator as described in section a.

rather gave slightly lower levels (see below). Despite this, they are being made available because they may prove useful for some inserted sequences. The strong predicted structure of the $3^{\prime}$ tail in these transcripts should form an independent RNA folding domain that is unlikely to interfere with folding of inserted sequence RNA.

\section{(b) Steady-state RNA expression}

The steady-state level of RNA produced from the SUP4 tRNA and RPR1 promoters was assessed by RNA blot hybridization and normalized to endogenous U6 RNA levels (Fig. 3 and Table II). RNA from both lowcopy (CEN6/ARS4) and high-copy $(2 \mu)$ vectors was examined either with no insert or with sequences corresponding to a 49-nt hairpin ribozyme (Hampel et al., 1989). RNA blots were probed in parallel with oligos to the recombinant $S U P 4$ leader (with $E c o$ RI site attached), to the recombinant $R P R 1$ leader, to the hairpin ribozyme insert, or to endogenous U6 RNA. The level of RNA transcripts per cell is given in Table II as a ratio of the leader hybridization signals to the endogenous U6 RNA signal. An cstimate of the absolute number of transcripts from the recombinant constructs can be obtained by multiplying these ratios by 1000 , a conservative estimate of the number of U6 RNA molecules per cell ( $\mathrm{Li}$ and Brow, 1993). With or without insert DNA, transcripts accumulate to a modest level from either the tRNA or $R P R 1$ promoter from the low-copy vector, and accumulate to a substantially higher level from the high-copy vector. It is interesting that the presence of a hairpin insert leads to higher levels of RNA accumulation in both the low-copy and high-copy versions (Fig. 3 and Table II). This might be due to stabilization against $3^{\prime}$ attack by the structured hairpin ribozyme or the strong stem/terminator that forms the end of the hairpin insert. To test this, low copy versions of both the SUP4 and $R P R 1$ promoters were tested in which a 6-bp stem with a tetraloop and a poly $(\mathrm{T})$ terminator was inserted without the hairpin sequences. These constructs did not accumulate at higher levels than RNA from the comparable clones without the $3^{\prime}$ stem terminator (see constructs designated $/ 3^{\prime} \mathrm{ST}$ in Table II). We conclude that the nature of the insert RNA affects RNA accumulation, but that an understanding of the nature of the stabilization will require a more systematic study.

The sizes of RNA products remain roughly the same from low- and high-copy vectors. The major RNA products in Fig. 3 correspond to expected sizes for the pri- 


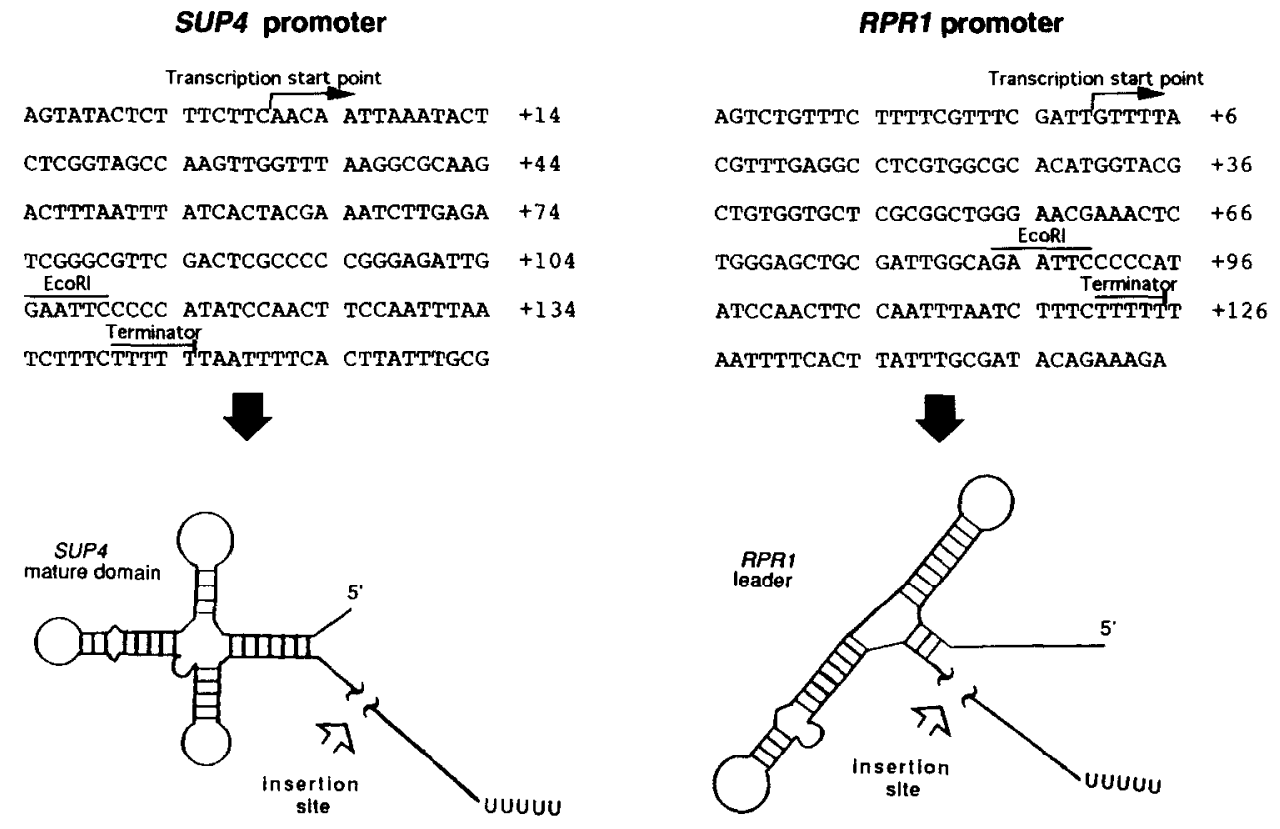

Fig. 2. Sequence and expected transcript structures for the pIIIEx pol III promoter constructs. The sequence of the SUP4 and RPRI promoter constructs is given from upstream from the transcription start points (marked with bent arrows) to downstream from the main expected termination points in the RPR1 terminator. The numbering represents bp after the transcription start point. The expected structure of the transcripts without inserts is shown below, $R P R 1$ leader structure is predicted according to Zuker (1989). An open arrowhead indicates the insertion position for sequences cloned into the EcoRI site. Hairpin ribozyme-encoding constructs contain the sequence 5'-AATTCNNNGTTGTAGAAGGCTACCAGAGAAACACACGTTGTGGTATATTACCTGGTATCGGGCGCAAGCCCGTTTTTTG inserted into the EcoRI site, where N represents nt that vary from clone to clone because the positions were deliberately randomized in the synthesis of the DNA oligos to be inserted. Note that the hairpin insert contains a $T_{6}$ transcription terminator and was expected to give efficient termination, but a minority of the transcripts appear to readthrough and terminate at the $R P R I$ terminator in the vector. In the case of the $/ 3$ 'ST constructs, transcripts would terminate with a 6 -bp stem and tetraloop immediately after the insertion site (not shown).

TABLE II

Normalization of pIIIEx vector transcripts to endogenous U6 RNA levels

\begin{tabular}{ll}
\hline Plasmid construct $^{\mathrm{a}}$ & Leader RNA/U6 $^{\mathbf{b}}$ \\
\hline pIIIEx316 tRNA & 0.18 \\
pIIIEx426 tRNA & 0.47 \\
pIIIEx316 tRNA hairpin & 0.40 \\
pIIIEx426 tRNA hairpin & 1.3 \\
pIIIEx316 tRNA/3'ST & 0.11 \\
& \\
pIIIEx316 RPR & 0.17 \\
pIIIEx426 RPR & 1.3 \\
pIIIEx316 RPR hairpin & 0.32 \\
pIIIEx426 RPR hairpin & 4.4 \\
pIIIEx316 RPR/3'ST & 0.09
\end{tabular}

${ }^{a}$ Growth of yeast containing listed plasmid constructs is described in Fig. 3 and Table I.

${ }^{b}$ Levels of RNA from plasmid constructs were determined by exposing the RNA blots in Fig. 3 on a Phosphorimager (Molecular Dynamics). The ratio of signal from pIIIEx plasmid transcripts to endogenous $U 6$ RNA is shown.

mary transcripts shown in Fig. 2. The largest RNAs in each lane are of appropriate sizes to represent termination at the vector $R P R 1$ terminator poly(T) site. In the case of the hairpin constructs, this means that the poly(T) sequence at the end of the hairpin insert does not act as a completely efficient terminator. For example, the expected tRNA transcript to the end of the hairpin stem/ terminator (pIIIEx316tRNA/hairpin or pIIIEx426tRNA/ hairpin) is $182 \mathrm{nt}$, but readthrough of this terminator to terminate instead at the $R P R 1$ terminator farther downstream gives a small amount of 224-nt RNA. RNA products shorter than the expected full-length transcript in each lane are of sizes consistent with trimming of the 5 , or $3^{\prime}$ ends of the transcripts at positions similar or identical to the processing intermediates that these leaders or the $R P R 1$ terminator region would normally undergo (Lee et al., 1991a).

In either SUP4 or RPR1 transcripts with inserted hairpin ribozyme sequences, there does not appear to be any accumulation of the leaders alone or detached hairpin sequences alone. It seems likely that this is due to the lack of any signals in the hairpin for cleaving it from the leaders, although we cannot rule out the possibility that such processing occurs and both leader and hairpin are subsequently destroyed. There is reason to believe, however, that if an RNA coding region containing its own processing signals is expressed from these vectors, that the leaders could be removed to leave the insert RNA product. It has been known for some time that in dimeric tRNA genc transcripts, the promoter is contained in the 


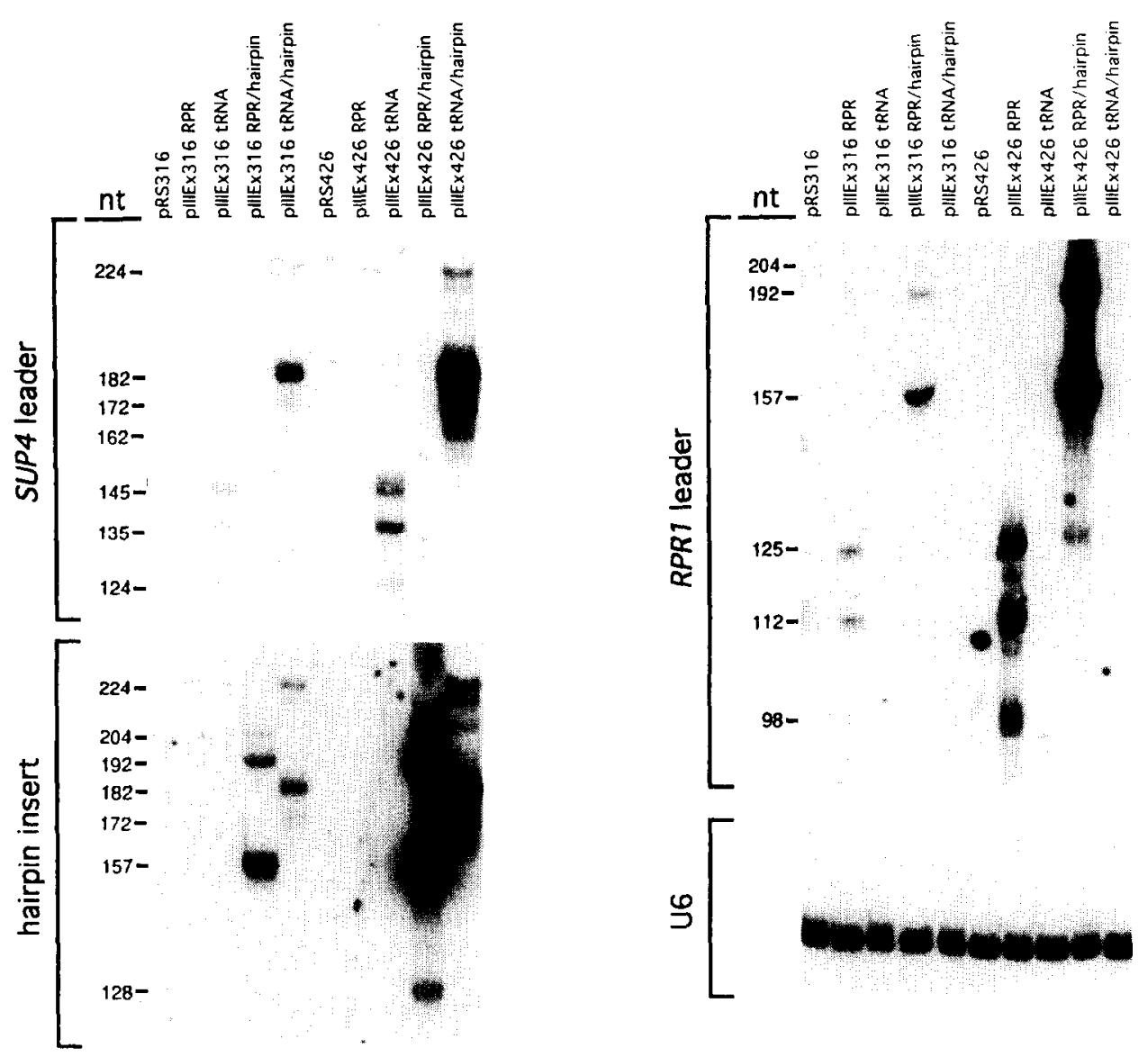

Fig. 3. RNA blot analysis of steady-state RNA levels. Cultures of S. cerevisiae strain, YM705 (MATa ura3-52 ade2-101 his3-4200 lys2-801 trp1-901 met $^{-}$) containing the indicated plasmids were grown in selective media and the RNA prepared as described (Kohrer and Domdey, 1991). Of each RNA, $10 \mu \mathrm{g}$ was subjected to electorphoresis in denaturing $10 \%$ polyacrylamide gels and electrotransferred to nylon membranes as described previously (Tranguch and Engelke, 1993). Duplicate membranes were probed witb the $5^{\prime}{ }^{32} \mathrm{P}$ oligos indicated to the left of each panel. These were complementary to either the RPR1 leader (5'-CGAATTCTGCCAATCGCAGCTCCCAG), the SUP4/EcoRI site junction (5'-GAATTCCAATCTCCCGG), the 3' half of the hairpin ribozyme insert (5'-CCGAATTCAAAAAACGGGCTTGCGCCCGATACCAGGTAATATACCACAACGTGT), or the +60 region of $S$. cerevisiae U6 RNA (5'-CCTTATGCAGGGGAACT); blots were exposed to X-ray film. The specific radioactivity of the probes was approx. the same but the exposure time was varied to best show minor bands: $30 \mathrm{~h}$ for $R P R 1$ leader, $24 \mathrm{~h}$ for the $S U P 4$ probe, $70 \mathrm{~h}$ for the hairpin insert probe and $26 \mathrm{~h}$ for the U6 probe. ${ }^{32} \mathrm{P}$-labelled RNA markers of known size (194, 147, 123 and $\left.111 \mathrm{nt}\right)$ were subjected to electrophoresis on the same gels to provide size estimates for the hybridizing species. The sizes of the major RNA products $( \pm 5 \mathrm{nt}$ ) are given to the left of the panels. Radioactivity in individual RNA species was determined using a Molecular Dynamics Phosphorimager and the levels of RNA produced from the expression constructs was normalized to the level of U6 RNA. The total RNA expressed from each construct is expressed as a ratio to the U6 RNA hybridization signal in Table II.

first tRNA coding region, but that the signals that specify cleavage between the two tRNAs reside in the second tRNA (Engelke et al., 1985). Information more directly pertinent to these pIIIEx vectors is also available. While we were most interested in expressing small, nonphysiological RNAs, others might desire to use these promoters to express RNAs normally found in yeast. The pIIIEx424 RPR vector has been used to express functional 5S rRNA as a substitute for the 5S rRNA from the endogenous gene. Roughly $75 \%$ of the normal $5 \mathrm{~S}$ rRNA level is cxpressed and the $R P R 1$ leader and terminator sequences are efficiently removed (S. Camier, personal communication). In this case the cell is clearly capable of recognizing the $5 \mathrm{~S}$ sequence in the context of the $R P R 1$ leader fusion and removing the extraneous 5 , and $3^{\prime}$ RNA. It is likely, then, that whether inserted sequences remain attached to the leaders and terminator sequences will depend on the nature of the insert.

\section{(c) Conclusions}

(1) Expression of small RNAs from two different RNA polymerase III promoters leads to stable accumulation of the RNA attached to the structured leaders. The presence of structured $3^{\prime}$ trailing sequences does not appear critical to levels of transcript accumulation.

(2) Levels of accumulation can be varied by using lowor high-copy-number expression vectors.

(3) Vectors are available with $H I S 3, T R P 1$ or $U R A 3$ markers. 


\section{ACKNOWLEDGEMENTS}

We thank P. Hieter for the gift of the pRS plasmids and $\mathrm{A}$. Tranguch for critical reading of the manuscript. This work was supported by research grants GM34869 and AI33263 to D.R.E. from the National Institutes of Health.

\section{REFERENCES}

Adeniyi-Jones, S., Romeo, P.H. and Zasloff, M.: Generation of long read-through transcripts in vivo and in vitro by deletion of $3^{\prime}$ termination and processing sequences in the human tRNA ${ }^{\text {meti }}$ gene. Nucleic Acids Res. 12 (1984) 1101-1115.

Cotten, M. and Birnstiel, M.L.: Ribozyme mediated destruction of RNA in vivo. EMBO J. 8 (1989) 3861-3866.

Engelke, D.R., Gegenheimer, P. and $\Lambda$ belson, J.: Nucleolytic processing of a tRNA ${ }^{\text {Arg }}{ }_{-}$RNA $^{\text {Asp }}$ dimeric precursor by a homologous component from Saccharomvces cerevisiae. J. Biol. Chem. 260 (1985) 1271-1279.

Goodman, H.M., Olson, M.V. and Hall, B.D.: Nucleotide sequence of a mutant eukaryotic gene: the yeast tyrosine-inserting ochre suppressor SUP4-o. Proc. Natl. Acad. Sci. USA 74 (1977) 5453-5457.

Hampcl, A., Tritz, R., Hicks, M. and Cruz, P.: 'Hairpin' catalytic RNA model: evidence for helices and sequence requirement for substrate RNA. Nucleic Acids Res. 18 (1990) 299-304.

Kjellin-Straby, K., Engelke, D.R. and Abelson, J.: Homologous in vitro transcription of linear DNA fragments containing the tRNA ${ }^{\text {Arg }}$ tRNA $^{\text {Asp }}$ gene pair from Saccharomyces cerevisiae. DNA 3 (1984) $167-171$.

Kohrer, K. and Domdey, H.: Preparation of high molecular weight RNA. Methods Enzymol. 194 (1991) 398-415.

Koski, R.A., Clarkson, S.G., Kurjan, J., Hall, B.D. and Smith, M.: Mutations of the yeast SUP4 tRNA ${ }^{\text {Tyr }}$ locus: transcription of the mutant genes in vitro. Cell 22 (1980) 415-425.

Kurjan, J., Hall, B.D., Gilam, S. and Smith, M.: Mutations at the yeast
SUP4 tRNA Tyr locus: DNA sequence changes in mutants lacking suppressor activity. Cell 20 (1980) 701-709.

Lee, J.-Y., Rohlman, C.E., Molony, L.A. and Engelke, D.R.: Characterization of RPRI, an essential gene encoding the RNA component of Saccharomyces cerevisiae nuclear R Nase P. Mol. Cell. Biol. 11 (1991a) 721-730.

Lee, J.-Y., Evans, C.F. and Engelke, D.R.: Expression of RNase P RNA in Saccharomyces cerevisiae is controlled by an unusual RNA polymerase III promoter. Proc. Natl. Acad. Sci. USA 88 (1991b) 6986-6990.

Li, Z. and Brow, D.A.: A rapid assay for quantitative detection of specific RNAs. Nucleic Acids Kes. 21 (1993) 4645-4646.

Pagan-Ramos, E., Tranguch, A.J., Kindelberger, D.W. and Engelke, D.R.: Replacement of the Saccharomyces cerevisiae RPR1 gene with heterologous RNase P RNA genes. Nucleic Acids Res. 22 (1994) 200-207.

Reyes, V.M., Newman, A. and Abelson, J.: Mutational analysis of the

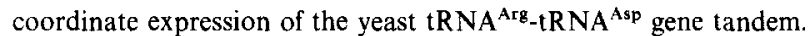
Mol. Cell. Biol. 6 (1986) 2436-2442.

Schmidt, O., Mao, R., Ogden, R., Beckmann, J., Sakano, H., Abelson, J. and Söll, D.: Dimeric tRNA precursors in yeast. Nature 287 (1980) 750-754.

Sikorski, R.S. and Hieter, P.: A system of shuttle vectors and yeast host strains designed for efficient manipulation of DNA in Saccharomyces cerevisiae. Genetics 122 (1989) 19-27.

Straby, K.B.: A yeast tRNA ${ }^{\text {Arg }}$ gene can act as promoter for a 5 ' flank efficient, non-transcribable tRNA ${ }^{\text {SUP6 }}$ gene to produce biologically active suppressor tRNA. Nucleic Acids Res. 16 (1988) 2841-2857.

Tabor, S. and Richardson, C.C.: DNA sequence analysis with a modified bacteriophage T7 DNA polymerase. Proc. Natl. Acad. Sci. USA 84 (1987) 4767-4771.

Tranguch, A.J. and Engelke, D.R.: Comparative structural analysis of nuclear RNase P RNAs from yeast. J. Biol. Chem. 268 (1993) 14045-14055.

Yu, M., Ojwang, J., Yamada, O., Hampel, A., Rapapport, J., Looney, D. and Wong-Staal, F.: A hairpin ribozyme inhibits expression of diverse strains of human immunodeficiency virus type 1. Proc. Natl. Acad. Sci. USA 90 (1993) 6340-6344.

Zuker, M.: On finding all suboptimal foldings of an RNA molecule Science 244 (1989) 48-52. 